\section{Las adolescentes frente a la decisión de interrumpir un embarazo en un contexto de aborto legal}

\author{
Adolescents faced with the decision to terminate \\ a pregnancy in a context of legal abortion
}

As adolescentes frente à decisão de interromper
uma gravidez em um contexto de aborto legal
Maria Luisa Ituarte 1

Alejandra López-Gómez 1

\section{Resumen}

El artículo presenta resultados de una investigación sobre las experiencias de las adolescentes que decidieron interrumpir su embarazo en el marco de los servicios legales de aborto en Montevideo (Uruguay), realizada entre los años 2016 y 2018. Su objetivo principal fue examinar el proceso de toma de decisión y el acceso al aborto legal, desde la perspectiva de las propias adolescentes. Ello implicó analizar circunstancias, motivaciones y personajes que influenciaron en la toma de la decisión y cómo ésta se tramitó en el servicio de salud. Se diseñó un estudio transversal de tipo exploratorio y descriptivo, basado en metodología cualitativa. La técnica principal que se utilizó para la recolección de la información fue la entrevista semidirigida, se entrevistó a 14 adolescentes entre 17 y 19 años de edad que interrumpieron voluntariamente un embarazo en los servicios públicos de salud. Los motivos para abortar dan cuenta del peso que tienen las razones socialmente aceptadas, incorporadas como motivos propios. Se observó que la madre juega un papel importante como referente en la decisión. Las adolescentes manifestaron tomar la decisión de abortar antes de haber concurrido al servicio de salud. Se destaca el papel positivo de la existencia de un marco legal que habilita servicios de aborto voluntario. Los resultados contribuyen a comprender los procesos que viven las adolescentes frente a una decisión vital difícil, el rol de la familia, en particular de la figura materna, el lugar que tiene el marco legal en la toma de la decisión, el peso del estigma y la sanción moral, y las expectativas respecto de la atención en servicios de salud.

Adolescencia; Aborto; Toma de Decisiones; Servicios de Salud

\section{Correspondencia}

M. L. Ituarte

Facultad de Psicología, Universidad de la República. Tristan Narvaja 1674, Montevideo - 11200, Uruguay. marialuisaituarte20@gmail.com

1 Facultad de Psicología, Universidad de la República, Montevideo, Uruguay. 


\section{Introducción}

El artículo presenta resultados de una investigación sobre las experiencias de adolescentes que decidieron interrumpir voluntariamente su embarazo en Montevideo, Uruguay, en el marco de los servicios legales de aborto disponibles en el país, a partir del año 2013.

El estudio tuvo como objetivo principal examinar el proceso de toma de decisión y el acceso a la interrupción voluntaria del embarazo en adolescentes montevideanas, que se asisten en servicios públicos de salud, desde la perspectiva de las propias adolescentes. Esto implicó analizar las circunstancias, motivaciones y personajes que influenciaron en la toma de la decisión y cómo ésta se tramitó en el servicio de salud.

El embarazo en adolescentes es considerado un problema de salud pública relevante a nivel mundial 1,2,3 y nacional 4 . Forma parte de determinantes vinculados a la exclusión, la vulnerabilidad y la falta de condiciones para el acceso a los derechos económicos, sociales y culturales 5 . Si bien existe abundante literatura a nivel regional y mundial sobre distintas dimensiones del embarazo no intencional en adolescentes, es significativamente menor la evidencia disponible sobre las experiencias de aborto en este grupo poblacional 6 .

Las decisiones sexuales y reproductivas en la adolescencia tienen consecuencias directas para la salud, pueden comprometer oportunidades académicas, sociales y laborales inmediatas; niveles de bienestar en la etapa adulta, oportunidades para sus hijos en el futuro y están determinadas por la distribución de los recursos económicos, educativos y sociales 1,7,8.

América Latina ocupa el segundo lugar en tasa de fecundidad adolescente con 66,4 por mil, muy por encima de Asia (42 por mil), América del Norte (27 por mil) y Europa (18 por mil) 9,10,11. A pesar de estar en una posición relativamente ventajosa respecto a la región, Uruguay se ubicó durante años como un caso de resistencia a la baja de la fecundidad adolescente, con una tasa de 59 por mil hacia 2014 12. Actualmente, esta tasa se ubica en 38 por mil, en el marco de un acelerado descenso de la tasa global de fecundidad en el país 13 . Sin embargo, la fecundidad adolescente es elevada, si se la compara con los países desarrollados 14 .

Las adolescentes que viven esta situación, no siempre disponen de las condiciones y garantías para acceder a un aborto seguro 15,16,17. En la mayoría de los países donde el aborto voluntario es ilegal, los servicios y la información sobre anticoncepción presentan importantes barreras de acceso para las adolescentes 11. Las evidencias disponibles indican que los/as adolescentes tienen comportamientos sexuales más seguros, si se les brinda herramientas e información en educación sexual y salud sexual y reproductiva 2,18,19,20. Los factores estructurales que pueden incidir en la ocurrencia de embarazo no intencional y en la toma de decisión de abortar son de tipo económico, social, cultural y subjetivo $18,21,22,23,24,25$.

En el caso uruguayo, la aprobación de la Ley no 18.987, de interrupción voluntaria del embarazo (IVE), del año 2012, implicó un cambio en el estatuto legal del aborto, posibilitando el acceso a esta práctica en el marco del Sistema Nacional Integrado de Salud (SNIS). Luego de años de debate social y parlamentario 26,27,28, Uruguay se sumó así a la corta lista de países de la región que habilita el aborto por sola voluntad de la mujer hasta las 12 semanas de gestación (14 semanas para el caso de violación). No se establece plazo para la interrupción cuando hay riesgo de vida o de salud para la mujer o malformación fetal incompatible con la vida extrauterina 29.

La Ley IVE y el decreto reglamentario del Ministerio de Salud (Decreto no 375/012 30) definen que la mujer debe transitar por cuarto instancias dentro del servicio de salud: una primer consulta médica (IVE 1), donde expresa su voluntad de abortar, una segunda consulta con un equipo multidisciplinario (IVE 2), luego de la cual la mujer está obligada a esperar cinco días con el objetivo de "reflexionar sobre la decisión"; una tercera consulta (IVE 3) en la que la mujer accede al aborto propiamente dicho en consulta con médico ginecólogo/a y una cuarta consulta de control y asesoramiento en anticoncepción (IVE 4) ${ }^{31}$. La ley habilita la objeción de conciencia de los profesionales de la salud, pero el servicio debe asegurar la atención. La ley establece la forma en que se recabará el consentimiento de las adolescentes. En casos de mujeres menores de 18 años, el médico tratante recabará el consentimiento para realizar la interrupción del embarazo ${ }^{32}$. De acuerdo a la edad de la adolescente y a la valoración de su autonomía, se propenderá a que las decisiones sean adoptadas por las propias adolescentes, y de ser posible, con la concurrencia de sus padres o referentes adultos de su confianza 33 . En caso de existir 
discrepancia entre la voluntad de la adolescente y la de los adultos a cargo, ésta podrá recurrir a un juez para laudar la situación, quien deberá velar por el derecho de la adolescente. En función de estos criterios, las adolescentes pueden transitar todo el proceso de IVE sin que se requiera necesariamente la participación de la familia.

Según los datos disponibles del Ministerio de Salud 34, entre 2013 y 2017, un 16\% del total de abortos anuales corresponden a adolescentes entre 15 y 19 años. Este porcentaje ha descendido para los años 2018 y 2019. La tasa anual de aborto por cada mil mujeres en edad reproductiva para 2018 fue de 11,39. Del total de mujeres que iniciaron el proceso de IVE, lo completaron el 94\%.

En este estudio buscamos conocer las experiencias subjetivas de las adolescentes que interrumpieron voluntariamente su embarazo. Para ello, nos ubicamos desde la perspectiva de la fenomenología social, que sostiene que lo que expresan los sujetos está relacionado con el contexto social, histórico y político que modela sus experiencias, centrándose en el mundo subjetivo de las personas 35 . En particular, nos propusimos explorar el proceso de la decisión de interrumpir el embarazo desde la perspectiva de las propias adolescentes, considerando los factores personales, familiares y sociales que incidieron en la decisión final.

\section{Método}

Se desarrolló un estudio transversal de tipo exploratorio y descriptivo a partir de un abordaje cualitativo. En este sentido, la realidad subjetiva en sí misma es objeto de estudio, donde se analizan los aspectos explícitos e implícitos, y se busca comprender cómo actúan y se comportan las personas, qué piensan, qué sienten, cuáles son sus actitudes en un ambiente particular 36,37. Se utilizó la entrevista semidirigida como técnica para la recolección de información, mediante la cual se buscó una aproximación empática a las experiencias de las adolescentes, a través de la exploración de sus perspectivas.

El universo de estudio incluyó a adolescentes residentes en Montevideo, entre las edades de 17 y 19 años, que vivieron la experiencia personal del aborto voluntario en los servicios públicos de salud. Para ello se realizó un acuerdo con la dirección de la Red de Atención Primaria Metropolitana de Administración de Servicios de Salud del Estado (RAP-ASSE) para involucrar a adolescentes elegibles, a través del contacto de los equipos de salud. Se definió una muestra intencional, por conveniencia, buscando incluir adolescentes con diferentes niveles educativos, con y sin hijos, y que hubiesen accedido a IVE en los 12 meses previos a la realización de la entrevista. El nivel educativo se tomó en base al rezago escolar, es decir la relación entre la edad de la adolescente y su escolaridad, en función de lo esperado para la edad. Se buscó incluir adolescentes vinculadas al sistema educativo con y sin rezago escolar y adolescentes que hubieran abandonado sus estudios. Se excluyeron a adolescentes con discapacidad psíquica y/o intelectual y adolescentes que hubieran abortado por fuera de los servicios legales de IVE (Cuadro 1).

Para la captación de las adolescentes se recurrió a la técnica de la bola de nieve y, para ello, se estableció contacto con los equipos de salud de los servicios de la RAP-ASSE y de otros servicios del SNIS, equipos técnicos vinculados a políticas sociales, organizaciones sociales, profesores de enseñanza media, entre otros.

La principal vía de captación se realizó a través de los equipos de IVE de los servicios de salud. Los profesionales hicieron el nexo con las adolescentes, tomaron contacto telefónico a los efectos de presentar las características de la investigación y les preguntaron si les interesaba participar. En el caso de aceptar, se les avisó que, desde el equipo de investigación, se pondrían en contacto para concretar una entrevista. El trabajo de recolección de información fue engorroso. Del total de adolescentes inicialmente contactadas por los equipos de salud, unas pocas accedieron a participar en el estudio. Cuando desde el equipo de investigación se las buscó contactar, se presentaron dificultades: la mayoría de las adolescentes no atendían los celulares o cambiaban de número, algunas aceptaron realizar la entrevista y se coordinó fecha y lugar, pero al volver a comunicarse no contestaban los teléfonos, y otras aceptaron participar si la entrevista era telefónica y no presencial. Esta situación exigió flexibilizar la propuesta inicial de realizar las entrevistas cara a cara, debido a la disposición mayoritaria de las adolescentes contactadas para participar en el estudio mediante una entrevista telefónica. En términos del desarrollo de la entrevista, en ambos casos fue posible completar la pauta prevista. El 
Cuadro 1

Datos de las adolescentes entrevistadas seleccionadas para la investigación.

\begin{tabular}{|c|c|c|c|c|c|}
\hline ENTREVISTA & SEUDÓNIMO & $\begin{array}{c}\text { EDAD CUANDO SE } \\
\text { HIZO IVE }\end{array}$ & NIVEL EDUCATIVO & CON O SIN HIJOS & TIPO DE ENTREVISTA \\
\hline 1 & María & 19 años & 4o año - liceo, con rezago escolar & Sin & Cara a cara \\
\hline 2 & Ana & 18 años & 1ㅇ año - facultad & $\operatorname{Sin}$ & Telefónica \\
\hline 3 & Bárbara & 18 años & 6o año - liceo & $\operatorname{Sin}$ & Telefónica \\
\hline 4 & Ximena & 19 años & 4o año - liceo, con rezago escolar & $\operatorname{Sin}$ & Telefónica \\
\hline 5 & Alejandra & 19 años & 5o año - liceo, con rezago escolar & $\operatorname{Sin}$ & Telefónica \\
\hline 6 & Victoria & 18 años & 6o año - liceo & $\operatorname{Sin}$ & Telefónica \\
\hline 7 & Sofía & 18 años & Abandonó sus estudios & 1 & Telefónica \\
\hline 8 & Analía & 19 años & 1ㅇ año - facultad de Derecho & $\operatorname{Sin}$ & Telefónica \\
\hline 9 & Macarena & 17 años & 4o año - liceo & $\operatorname{Sin}$ & Telefónica \\
\hline 10 & Carolina & 18 años & 6o año - liceo & $\operatorname{Sin}$ & Telefónica \\
\hline 11 & Candelaria & 19 años & 6o año - liceo & $\operatorname{Sin}$ & Telefónica \\
\hline 12 & Giselle & 19 años & Abandonó sus estudios & 1 & Cara a cara \\
\hline 13 & Laura & 18 años & Abandonó sus estudios & $\operatorname{Sin}$ & Cara a cara \\
\hline 14 & Gloria & 19 años & 1ㅇ año - facultad de Arquitectura & $\operatorname{Sin}$ & Cara a cara \\
\hline
\end{tabular}

IVE: interrupción voluntaria del embarazo.

análisis de los relatos no arrojó diferencias importantes derivadas de la modalidad de entrevista. Por otra parte, algunas de las que aceptaron realizar las entrevistas parecían hacerlo como modo de agradecimiento al buen trato de los profesionales que las atendieron en los servicios de salud. Si bien se intentó controlar este sesgo, ampliando las estrategias de captación de adolescentes elegibles, ello no resultó posible y es, por tanto, una de las limitaciones del estudio.

El trabajo de recolección de datos tuvo lugar entre los meses de abril y octubre del 2017 y se realizaron 14 entrevistas a las adolescentes que aceptaron participar previa información sobre los objetivos de la investigación. Se les informó que la entrevista era anónima y confidencial, y que no se la identificaría en ningún momento del proceso, incluida la difusión de sus resultados. Durante el transcurso de las entrevistas se prestó especial atención a las reacciones o repercusiones que las mismas pudieran ocasionar en las participantes, en el sentido de provocar algún tipo de malestar emocional. Si bien las reacciones fueron diversas, no fue necesaria la derivación a consulta de apoyo psicológico. Las entrevistas tuvieron una duración entre 45-60 minutos y fueron grabadas con el consentimiento de las adolescentes. Del total de entrevistas realizadas, cuatro fueron cara a cara en una sala acondicionada para entrevistas de investigación del local de la Universidad y 10 fueron telefónicas. Posteriormente, las entrevistas fueron transcritas y formateadas para su posterior codificación, sistematización y análisis, siguiendo los procedimientos del análisis de contenido.

El proyecto tuvo el aval del Comité de Ética en Investigación de la Facultad de Psicología de la Universidad de la República y de la Comisión de Investigación de RAP-ASSE. Se solicitó el asentimiento a las participantes, de acuerdo a lo previsto por el Decreto no 379/008 del Ministerio de Salud 38 y, a solicitud de los comités de ética, se requirió el consentimiento de los padres o representantes, en caso de que fuera necesario. 


\section{Resultados y discusión}

Se entrevistaron a 14 adolescentes entre 17 y 19 años residentes en Montevideo y usuarias de servicios públicos de salud de la RAP-ASSE. Tres de las adolescentes entrevistadas habían abandonado sus estudios y dos de ellas tenían hijos; la mayoría se encontraban cursando enseñanza media o bachillerato, aunque tres de ellas estaban desfasadas en el calendario escolar y tres estaban cursando carreras universitarias. Todas tenían cobertura de salud en el sector público de la RAP-ASSE. El nivel educativo y la cobertura de salud (pública) pueden considerarse proxy de nivel socioeconómico. Desde el punto de vista de autoidentificación étnico-racial, todas se definieron como blancas. En términos de integración del hogar, las adolescentes entrevistadas vivían con sus familias, y ninguna tenía pareja conviviente en ese momento.

Se definió una lista de códigos de acuerdo a la pauta de entrevista que fue complementada de manera inductiva con códigos emergentes. La codificación y sistematización del corpus empírico permitió identificar las principales dimensiones de análisis, las cuales organizan la presentación de los resultados en cuatro apartados: reacciones emocionales frente a la noticia del embarazo; motivos para interrumpirlo; influencias en la decisión; acceso al aborto en el servicio de salud. Se analizó la relación entre la demanda y la oferta de atención, a partir de las experiencias de las propias adolescentes.

\section{Reacciones frente al embarazo}

Las adolescentes contaron que el embarazo fue inesperado, se trató de un acontecimiento que irrumpió en sus vidas y les generó desconcierto y sentimientos de rechazo.

Entrevistadora: "¿Y qué sentiste ante la posibilidad de estar embarazada?”.

Ana (18 años): "Me sentí mal, obviamente. Me sentí con esa impotencia de decir 'ipor qué?'. Obviamente no era lo que deseaba".

Entrevistadora: “¿Cuál fue tu primera reacción?".

Alejandra (19 años): "No, me puse a llorar".

Entrevistadora: “¿Y qué fue lo primero que pensaste?”.

Alejandra (19 años): "Que me lo quería sacar. Que no quería tenerlo".

Del relato de las adolescentes se desprende que se trató de embarazos no intencionales, producto del uso inconsistente de método anticonceptivo o de la dificultad de su negociación con la pareja sexual. Los estudios disponibles en el país registran que, en general, las adolescentes tienen conocimiento de los métodos anticonceptivos ${ }^{39}$, sin embargo, se registra a nivel nacional una alta incidencia de embarazos no intencionales en este grupo de edad ${ }^{40}$. A su vez, varios estudios coinciden en afirmar que el conocimiento de los métodos no asegura un uso adecuado y consistente 4,41,42. El relato de esta adolescente ilustra el desconocimiento del funcionamiento de los métodos y el uso inadecuado de la anticoncepción de emergencia.

Entrevistadora: “'Y estabas usando algún método anticonceptivo?".

Candelaria (19 años): "Justo hacía como un mes, un mes y medio que habia dejado las pastillas anticonceptivas y hubo un momento después, cuando me enteré de cuántas semanas estaba, yo no me había protegido con mi pareja, pero había tomado una pastilla de emergencia antes de las 48 horas, que en realidad son 72, pero yo las tomé antes de las 48 y se ve que falló, no sé".

En un principio, las adolescentes negaron el embarazo, a pesar de haberse percatado del retraso menstrual, razón por la cual demoraron en confirmarlo o descartarlo.

Entrevistadora: "¿En qué momento pensaste o sospechaste que podrías estar embarazada?".

Gloria (19 años): "En realidad primero lo sospeché por, o sea me enteré tarde... porque no me había dado cuenta, en un principio, la dejé pasar y bueno... después de que pasaron como 3 semanas ahí se me dio por hacerme el test y bueno...".

Entrevistadora: “¿Cómo te sentiste ante esa posibilidad de estar embarazada?".

Ana (18 años): "Desde un principio estaba convencida de que no... Nunca quise, digamos. No tenía ni idea de cómo era ser madre y no tenía ganas de ser madre. Nunca se me pasó por la cabeza tener un hijo a tan corta edad y menos estudiando".

La demora en "darse cuenta" del embarazo es un rasgo propio en la adolescencia, asociado a mecanismos psicológicos de negación, a las dificultades para valorar las consecuencias a futuro y a la 
omnipotencia. Dicha demora genera que las adolescentes acudan al servicio de salud más próximo a las 12 semanas de gestación. Aquí se pone en juego la vivencia subjetiva del tiempo, el cual es percibido como ilimitado por estas adolescentes, sin tener en cuenta las consecuencias futuras de no contar con el tiempo que define la ley para acceder a la interrupción del embarazo en el servicio de salud 2.

Se trata de una actitud basada en una racionalidad mágica que no permite prever las consecuencias que tienen los comportamientos. Sin embargo, limitarnos solo a este componente, no permite dar cuenta de un conjunto de aspectos que operan "sobre las personas" 43,44, como, por ejemplo, la falta de educación sexual de calidad, el temor a la reacción de la familia, entre otras.

\section{Motivos para interrumpir el embarazo}

Desde una perspectiva fenomenológica de la experiencia subjetiva, la acción de los individuos se puede interpretar según los motivos, a los cuales clasifica en motivos porque y motivos para. Los motivos porque refieren a las experiencias y determinaciones pasadas del sujeto que lo llevaron a actuar de tal manera. Los motivos para aluden a la acción orientada al futuro, es decir al estado de cosas u objetivos que se pretenden alcanzar con determinada acción 35 .

Los principales motivos para decidir interrumpir el embarazo que manifestaron las entrevistadas fueron su edad temprana, la imposibilidad económica (no trabajaban y dependían económicamente de sus padres), se encontraban estudiando (no querían dejar de estudiar o posponer sus estudios), no se sentían preparadas emocional y psicológicamente para tener un hijo, se encontraban en una relación amorosa reciente o no estable y, por último, porque el embarazo "no era algo deseado". En su mayoría se trata de motivos para, que refieren a lo que las adolescentes pretenden alcanzar con la acción de interrumpir el embarazo, entre otros, continuar con los estudios, no proyectar un vínculo de pareja a futuro, no hacerse cargo del cuidado de un hijo/a. Estos resultados coinciden con lo planteado por otros estudios a nivel regional y mundial 45,46,47,48,49,50.

Entrevistadora: “¿Y por qué te planteaste interrumpir el embarazo?”.

Carolina (18 años): "Primero que nada el tema de la edad, los dos estamos estudiando, sería bastante difícil y también por temas económicos porque por ejemplo a mí todavía me siguen manteniendo mis padres y no sería correcto y también por temas psicológicos, creo que todavía no estamos preparados psicológicamente para tener un hijo".

María (19 años): "Primero por un tema de edad, de economía, porque yo estaba estudiando nada más, no tenía trabajo, dependía de mis padres en este caso y no me sentía preparada emocional ni... con la relación tampoco, era una relación bastante reciente, estábamos hacía por lo menos siete meses pero realmente no quería imponerme una relación por tener un hijo en común, y ya te digo, no contaba con los medios para poder mantener a un niño en ese caso".

Entrevistadora: “¿Cuáles fueron las razones que te planteaste interrumpir el embarazo?”.

Macarena (17 años): "Por el tema de que quiero seguir estudiando y todavía no sé lo que quiero para mi vida y menos voy a saber para un bebé".

La continuación de un embarazo es percibida como una limitación de sus posibilidades a futuro, de desarrollo e inserción en el mercado laboral y, en ese sentido, la maternidad es vivida como un problema. Las representaciones que pueden tener las adolescentes en cuanto a la función materna se pueden relacionar con cuatro aspectos: engendrar, concebir, parir y criar. Para estas adolescentes, la función materna implica la responsabilidad del cuidado y bienestar del hijo/a, la alta disponibilidad afectiva y la postergación de las necesidades y deseos propios. En este sentido, uno de los principales motivos para abortar era no sentirse preparadas para asumir esta responsabilidad, la cual es vivida como una exigencia social que sienten no poder sostener 51 .

\section{Influencias en la toma de la decisión}

Se buscó indagar acerca del papel que jugaron personajes próximos en la vida de estas adolescentes en la toma de la decisión, en particular la madre y/o familia, pareja, amigas. Las adolescentes expresaron no haberse sentido presionadas por su entorno frente a la decisión, sea para continuar o para interrumpir el embarazo. 
Una de las condiciones importantes para procesar una decisión vital difícil como continuar o interrumpir un embarazo, son los recursos familiares de apoyo 17. Estos recursos, cuando están disponibles juegan un papel protector de la salud y/o amortiguador de procesos de malestar o padecimiento 35 .

Entrevistadora: “Qué cosas sentís que te ayudaron a vivir ese momento y qué cosas no te ayudaron?".

María (19 años): "Ya te digo, principalmente, lo que me ayudó a pasarlo mejor o bueno, lo menos mal posible fue el apoyo de mi familia, mis seres queridos y la atención tan rápida y sobre todo tan comprensiva de los médicos y demás (...) Ahí dije: 'es el momento de hablarlo', lo hablé con mis hermanas que son las más confidentes y me apoyaron para contarlo a mi familia, a mi madre, mi padre y bueno, mi pareja en realidad fue la última en enterarse porque sabía yo que era el que más iba a tener problemas para aceptar mi decisión de interrumpir el embarazo, y fue una situación bastante complicada, porque él no estaba muy de acuerdo pero supo entender mi postura, y comenzó el proceso de las consultas y eso con los especialistas".

En particular, las madres juegan un papel importante en la decisión de sus hijas, en la medida que su opinión es importante para las adolescentes. Habitualmente, la madre ofrece un modelo de identificación a la adolescente, lo que la ubica en tanto figura de peso en la toma de decisiones 52 . La literatura sugiere que la salud sexual y reproductiva de las adolescentes puede ser particularmente influenciada por sus madres, ya que son las principales fuentes de comunicación sobre sexualidad con sus hijas 53 .

Entrevistadora: “¿Y la opinión de tu mamá influyó en tu toma de decisión?".

Candelaria (19 años): "En realidad ella me dijo que hiciera lo que quisiera que ella me iba a apoyar pero me aconsejó que soy joven, todo lo que te decía, que estudio y eso, pero más allá de que ella me hubiera dicho ay qué bueno que estaría, ta yo tomo sus consejos pero no es algo que por lo que ella me diga yo decida. O sea, yo desde un principio ya sabía que no, que no iba a tener, o sea, yo tomo sus consejos y ta, pero la decisión fue completamente mía".

Así como disponer del apoyo familiar es una fuente de bienestar y favorece la autoafirmación en las propias decisiones, el miedo a la reacción del entorno es fuente de angustia. Algunas adolescentes tomaron la decisión de no compartir la situación con su familia como forma de evitar el conflicto, y por el temor a la expulsión del hogar, a la sanción moral o física, al rechazo parental. Esto significa que, si los vínculos familiares son vividos como protectores, la situación es compartida en primer lugar en ese espacio. Por el contrario, si el espacio familiar es vivido con temor al rechazo, la situación es ocultada y, por tanto, la familia lejos de ser un factor protector o de apoyo social se transforma en un factor de riesgo de exposición al daño.

Entrevistadora: “¿Y se lo contaste a alguien más?".

Analía (19 años): "A la mamá de él y al hermano".

Entrevistadora: "¿Y a tu mamá o a algún familiar tuyo se lo contaste?”.

Analía (19 años): "No, no, porque, están en contra de eso, aparte son, por así decirlo, religiosos. Y ahí sería medio conflictivo para mí también, enfrentarlo con ellos. No sé cómo reaccionaría".

Entrevistadora: "¿Y tu familia se enteró sobre esto?".

Carolina (18 años): "No, no. No, simplemente no sé, por miedo".

Entrevistadora: “'Miedo de lo que te puedan decir?”.

Carolina (18 años): "Claro, por los prejuicios que hay todavía sobre el tema, sigue siendo bastante tabú entonces... Aunque está legalizado sigue siendo tabú".

Para algunas adolescentes, el aborto es una experiencia socialmente estigmatizada y estigmatizante. A pesar del cambio legal, deben enfrentar el peso del cuestionamiento social a una decisión que se considera irresponsable y consecuencia de una vida sexual no protegida.

Así como la familia, y en particular la madre, parecen jugar un papel importante en el proceso de decisión, no sucede lo mismo con los/as pares. Las adolescentes no acudieron a amigas/os para compartir su situación. Sumter et al. 54 sostienen que las adolescentes mujeres tienen mayor resistencia a la influencia de sus amigas en comparación a lo que sucede con los adolescentes varones, quienes son más propensos a la influencia de sus pares. Los autores lo explican en términos de maduración psicosocial, ya que las adolescentes presentan mayor autorregulación, responsabilidad y mayor grado de conciencia de sus actos, por lo cual a mayor maduración psicosocial menor es la susceptibilidad a la influencia de sus pares.

Dado que, en todos los casos, el embarazo tuvo lugar en el marco de un vínculo afectivo de noviazgo o amoroso reciente, se buscó explorar la participación de estos varones en el proceso de toma de decisión. Para algunas adolescentes, la decisión fue de mutuo acuerdo, en otras -como ya hemos refe- 
rido- fue una decisión controvertida dentro de la pareja. Las adolescentes expresaron que no tomaron la decisión bajo presión, por temor al abandono o el desamor de sus parejas, para evitar situaciones de violencia o para satisfacer un deseo de sus parejas.

Entrevistadora: "¿Y se lo contaste a tu pareja?".

Analía (19 años): "Sí, se lo conté. Cuando se lo conté yo no tenía todavía una resolución, como quién dice, determinada, sino que se lo conté y ahí fue cuando me dijo que tampoco podía. Entonces como que ta!...”.

Entrevistadora: “Entonces ¿la decisión la tomaste vos sola o fue con él?".

Gloria (19 años): "No, después de que lo hablamos, fue una decisión que yo plantee y que él la aceptó. Y lo terminamos aceptando los dos digamos".

Para algunas adolescentes, el aborto es una experiencia socialmente estigmatizante, y ese estigma se incorpora subjetivamente. A pesar del cambio legal, deben enfrentar el peso del (auto) cuestionamiento social por una situación que consideran fruto de la irresponsabilidad. Deben enfrentarse a sus actitudes negativas previas sobre el aborto y colocarse en los zapatos de aquellas mujeres que decidieron abortar.

Entrevistadora: "¿Y por qué crees que las personas pueden juzgarte frente a un embarazo no deseado?".

Gisele (19 años): "Porque yo siempre decía que yo nunca iba a abortar, nunca iba a abortar y me tocó a mí. Y ta, entonces me quedé callada y no dije nada a nadie".

Entrevistadora: “'Tú antes tenías la idea, tú qué pensabas acerca del aborto?".

Gisele (19 años): "No, yo estaba en contra del aborto. Pero ta, nunca pensé que me iba a tocar a mí".

\section{El papel del servicio de salud en la toma de decisión}

Se buscó conocer el papel del servicio de salud en la toma de la decisión. La mayoría de las adolescentes manifestaron que su decisión fue tomada previamente a la consulta en el centro de salud.

Las adolescentes contaban con información sobre el marco legal y los procedimientos a seguir con anterioridad a la consulta con el centro de salud. Si bien en algunos casos era escasa o imprecisa, en general, la habían obtenido a través de familiares o de la institución educativa a la cual asistían. La disponibilidad de servicios de IVE fue un factor importante que influyó positivamente en la decisión de interrumpir. Como dice María (19 años), la ley le daba "seguridad", "me sentía amparada, que no iba a ser una búsqueda a oscuras, a tientas...".

En general, las adolescentes evaluaron de manera negativa el plazo de cinco días de reflexión que establece la ley luego de la segunda consulta (IVE 2, con el equipo interdisciplinario), ya que es un tiempo de espera que les produjo mayor angustia y ansiedad. En tal sentido, las adolescentes expresaron que esta situación provoca una demora en la concreción del "alivio" que se aspira tener al terminar cuanto antes con el proceso de aborto.

Entrevistadora: “Cómo fueron esos 5 días de reflexión?”.

Gisele (19 años): "Fue horrible. Desearía que ya pasaran esos 5 días. Yo le dije al ginecólogo que yo no quería esos 5 días, si yo ya estaba decidida a que me lo iba a sacar y él me dijo que no, que me tenía que dar 5 días sí o sî".

Los centros de salud fueron percibidos como lugares confiables y seguros, donde los/as profesionales les brindaron buena atención y cuidado. Destacaron la sensibilidad, la capacidad de escucha, comprensión y la confianza que les generó el equipo multidisciplinario, siendo factores protectores y habilitantes para las adolescentes. En este punto, varios estudios publicados en los últimos años en Latinoamérica analizan las actitudes, valores y prácticas de los/as profesionales frente a las mujeres en situación de aborto y su influencia en el acceso a un aborto seguro 55 . Algunos destacan el peso del estigma del aborto 56,57 en las prácticas profesionales, expresado en las resistencias de los/as profesionales, en el plano de las convicciones morales, los sistemas de valores y los estereotipos de género.

La legalidad de la práctica y la disponibilidad de servicios en el país es un factor clave en la decisión de abortar, en tanto las adolescentes manifestaron sentir mayor protección frente a la situación. Nuestras entrevistadas no manifestaron temor al rechazo de los profesionales de la salud ni haber vivido situaciones de maltrato en el servicio. Por el contrario, expresaron gratitud por la atención recibida. Este punto amerita ser estudiado con mayor profundidad en futuras investigaciones. Por un lado, la calidad de atención en los servicios de IVE en Uruguay ha sido analizado parcialmente, y no se dispone de evidencias sobre la calidad de atención a población adolescente. En segundo lugar, 
el estudio de la calidad de atención, desde la perspectiva de las propias usuarias, tiene complejidades derivadas de los sesgos de satisfacción, producto de las relaciones desiguales de poder que se establece entre usuarias y profesionales. Más aún si se considera el desbalance y la situación de vulnerabilidad que atraviesan las adolescentes en situación de aborto. Por tanto, los resultados que hemos obtenido pueden considerarse como una aproximación a las valoraciones de las adolescentes sobre la atención recibida en la consulta IVE 2 y el papel que jugaron los servicios en la decisión de interrumpir el embarazo, y no como indicador de la calidad de atención de los servicios.

\section{Conclusiones}

El estudio aporta conocimiento sobre los factores que inciden en la toma de decisión de interrumpir un embarazo en adolescentes entre 17 y 19 años en los servicios de salud públicos de Montevideo. El aborto es considerado un proceso con un antes, un durante y un después, así como un evento significativo en la vida de las adolescentes que debe ser analizado en el contexto en el cual tiene lugar. En todos los casos se trató de embarazos no intencionales, relacionados con el uso inconsistente de métodos anticonceptivos. El análisis de los motivos para abortar da cuenta del peso que tienen las razones socialmente aceptadas, incorporadas como motivos propios. Los resultados contribuyen a comprender los procesos que viven las adolescentes frente a una decisión vital difícil, el papel de la familia y en particular de la figura materna, el lugar que tiene el marco legal en la toma de la decisión, el peso del estigma y la sanción moral, las expectativas respecto de la atención en los servicios de salud. Las adolescentes concurren al servicio de salud con la decisión tomada, y buscan en los profesionales comprensión y apoyo para efectivizarla. Los resultados obtenidos pueden ser de utilidad para la implementación de servicios de aborto legal dirigidos a población adolescente, en particular por aportar evidencias sobre algunos elementos clave en el proceso de toma de decisión, desde la perspectiva de las propias adolescentes. El estudio tiene limitaciones en: el perfil de la muestra obtenida, que tiene el sesgo de haber sido captada por los equipos de salud de los servicios públicos que las atendieron; haber entrevistado mayormente a adolescentes de 18 y 19 años, y haber realizado entrevistas cara a cara y entrevistas telefónicas por imposibilidad de realizarlas todas de manera presencial, en consideración a la solicitud o disposición de las adolescentes. 


\section{Colaboradores}

M. L. Ituarte contribuyó en el trabajo de recolección de datos del proyecto, análisis y escritura del artículo. A. López-Gómez contribuyó en el análisis y discusión del material empírico y revisó la escritura final del manuscrito.

\section{Informaciones adicionales}

ORCID: Maria Luisa Ituarte (0000-0003-47754224); Alejandra López-Gómez (0000-0001-99757297).

\section{Agradecimientos}

Agradecemos a las adolescentes que aceptaron participar en el estudio y a los/as profesionales de los servicios de salud de la Red de Atención Primaria Metropolitana de la Administración de los Servicios de Salud del Estado (RAP-ASSE) que ofrecieron su colaboración. La investigación se realizó en el marco de la obtención del título de Magister en Psicología Social de la Facultad de Psicología de la Universidad de la República (Uruguay) y contó con el financiamiento de la Agencia Nacional de Investigación e Innovación (ANII).

\section{Referencias}

1. World Health Organization. Global Accelerated Action for the Health of Adolescents (AA-HA!): guidance to support country implementation. Geneva: World Health Organization; 2017.

2. Cherry AL, Dillon ME. International handbook of adolescent pregnancy. Medical, psychosocial, and public health responses. New York: Springer; 2014.

3. United Nations Population Fund. State of the world population: motherhood in childhood facing the challenge of adolescent pregnancy. New York: United Nations Population Fund; 2017.

4. López A, Varela C. Maternidad en adolescentes y desigualdad social en Uruguay. Montevideo: Universidad de la República/Fondo de Población de las Naciones Unidas; 2016.

5. Chandra-Mouli V, Akwara E, Engel D, Plessons M, Asnake M, Mehra S, et al. Progress in adolescent sexual and reproductive health and rights globally between 1990 and 2016: what progress has been made, what contributed to this, and what are the implications for the future? Sex Reprod Health Matters 2020; 28:1741495

6. López-Gómez A. Mujeres y aborto. El papel de las condiciones legales y sociales en las trayectorias y experiencias subjetivas de las mujeres frente al aborto inducido. In: Ramos S, compiladora. Investigación sobre aborto en América Latina y el Caribe. Una agenda renovada para informar política pública e incidencia. Buenos Aires: Centro de Estudios de Estado y Sociedad/Population Council/Consorcio Latinoamericano contra el Aborto Inseguro; 2015. p. 19-40.

7. Córdova Pozo K, Chandra-Mouli V, Decat P, Nelson E, De Meyer S, Jaruseviciene L, et al. Improving adolescent sexual and reproductive health in Latin America: reflections from an International Congress. Reprod Health 2015; 12:11.

8. World Health Organization. WHO guidelines on preventing early pregnancy and poor reproductive outcomes among adolescents in developing countries. Geneva: World Health Organization; 2011.

9. Pan American Health Organization. Accelerating progress toward the reduction of adolescent pregnancy in Latin America and the $\mathrm{Ca}$ ribbean.Washington DC: Pan American Health Organization; 2016. (Report of a Technical Consultation).

10. Rodríguez J. Deseabilidad y planificación de la fecundidad adolescente en América Latina y el Caribe: tendencias y patrones emergentes. Notas de Población 2017; (104):119-44.

11. Reina MF, Castelo-Branco C. Teenage pregnancy: a Latin-American concern. Obstetrics and Gynecology Research 2018; 1:85-93. 
12. Varela C, Tenenbaum M, Lara C. Fecundidad adolescente en el Uruguay: ¿la pobreza como umbral de resistencia al descenso. In: Cavenaghi S, Cabella W, editores. Comportamiento reproductivo y fecundidad en América Latina: una agenda inconclusa. Río de Janeiro: Asociación Latinoamericana de Poblaciones; 2014. p. 185-206. (Serie de Investigaciones, 3).

13. Ministerio de Salud Pública. Estrategia nacional e intersectorial de prevención del embarazo no intencional en adolescentes. Montevideo: Ministerio de Salud Pública/Fondo de Población de las Naciones Unidas; 2020.

14. Fondo de Población de las Naciones Unidas. Descenso acelerado de la fecundidad en Uruguay entre 2015 y 2018. Tres estudios para su análisis. Montevideo: Fondo de Población de las Naciones Unidas; 2019.

15. Diniz D, Medeiros M. Aborto no Brasil: uma pesquisa domiciliar com técnica de urna. Ciênc Saúde Colet 2010; 15:959-66.

16. Diniz D, Medeiros M. Itinerários e métodos do aborto ilegal em cinco capitais brasileiras. Ciênc Saúde Colet 2012; 17:1671-81.

17. Heilborn ML, Cabral CS, Brandão ER, Cordeiro F, Azize RL. Gravidez imprevista e aborto no Rio de Janeiro, Brasil: gênero e geração nos processos decisórios. Sex Salud Soc (Rio J.) 2012; (12):224-57.

18. Rivera S. Embarazo en adolescentes. Aspectos de tipo social, psicológico y cultural. http:// issuu.com $/ \mathrm{mijor} /$ docs/embarazo-en-adoles centes (accedido el 05/Jul/2020).

19. García Baños L, López Baños L, Alonso Sicilia M. La bioética y el derecho de las adolescentes en el aborto. Rev Cuba Obstet Ginecol 2013; 39:404-12.

20. Fondo de Población de las Naciones Unidas. Sistematización de evidencias científicas sobre la educación integral de la sexualidad. Panamá: Fondo de Población de las Naciones Unidas/ Oficina Regional de UNICEF para América Latina y el Caribe; 2018.

21. Martín L, Reyes Z. Conducta sexual, embarazo y aborto en la adolescencia. Un enfoque integral de promoción de salud. Rev Cuba Salud Pública 2003; 29:183-7.

22. Collado ME, Alva R, Villa L, López E, González de León D, Schiavon R. Embarazo no deseado y aborto en adolescentes: un reto y una responsabilidad colectiva. Género y Salud en Cifras 2008; 6:17-30.

23. Álvarez L, Salomón N. El aborto en adolescentes en un contexto legal. Rev Cuba Salud Pública $2012 ; 38: 45-53$.

24. Flores JA. Aborto en la adolescencia. Impacto ético y social. La Habana: Medisur; 2015.

25. Malcolm AL, Rodríguez AR. Aborto provocado en la adolescencia. Multimed (Granma) 2015; 19(4). http://www.revmultimed.sld.cu/ index.php/mtm/article/view/365.

26. Uruguay. Ley no 9.763. Aborto. Diario Oficial 1938; 28 ene.
27. Abracinskas L, López-Gómez A, coordinadoras. Aborto en debate: dilemas y desafíos del Uruguay democrático. Proceso político y social 2001-2004. Montevideo: Mujer y Salud en Uruguay; 2007.

28. Johnson N, López A, Sapriza G, Castro A, Arribeltz G. (Des)penalización del aborto en Uruguay: prácticas, actores y discursos. Abordaje interdisciplinario sobre una realidad compleja. Montevideo: Universidad de la República/Comisión Sectorial de Investigación Científica; 2011.

29. Uruguay. Ley no 18.987. Interrupción voluntaria del embarazo. Diario Oficial 2012; 30 oct.

30. Uruguay. Decreto no 375/012. Reglamentación de la ley sobre interrupción voluntaria del embarazo. Ley del aborto. Diario Oficial 2012; 29 nov.

31. López A, Couto M, Píriz G, Monza A, Ituarte M, Abracinskas L. Servicios legales de interrupción voluntaria del embarazo en Uruguay. Estrategias de los servicios públicos del primer nivel de atención de Montevideo y área Metropolitana. Salud Pública Méx 2017; 59:57782.

32. Carril E, López A. Entre el alivio y el dolor. Mujeres, aborto voluntario y subjetividad. Montevideo: Trilce; 2008.

33. Uruguay. Ley no 18.426. Ley sobre salud sexual y reproductiva. Diario Oficial 2008; 10 dic.

34. Ministerio de Salud Pública. Tendencias recientes de la fecundidad, natalidad, mortalidad infantil en el Uruguay. Montevideo: Ministerio de Salud Pública; 2018.

35. Castro R. Teoría social y salud. México DF: Editorial Lugar; 2011.

36. Sampieri R, Collado C, Lucio P. El proceso de investigación y los enfoques cuantitativo y cualitativo hacia un modelo integral. In: Sampieri $\mathrm{R}$, Collado C, Lucio P, editores. Metodología de la investigación. México DF: McGraw-Hill Interamericana; 2003. p. 14-5.

37. Taylor S, Bogdan R. Introducción. Ir hacia la gente. In: Taylor S, Bogdan R, editores. Introducción a los métodos cualitativos de investigación. Barcelona: Ediciones Paidós Ibérica; 1987. p. 19-25.

38. Uruguay. Decreto no 379/008. Diario Oficial 2008; 14 ago.

39. Amorín D. Apuntes para una posible psicología evolutiva. Montevideo: Psicolibros/Waslala; 2008.

40. Observatorio Nacional en Género y Salud Sexual y Reproductiva. Estudio 2011-2012: necesidades y demandas en salud sexual y reproductiva en mujeres adolescentes uruguayas. Montevideo: Mujer y Salud en Uruguay; 2013.

41. De Dios Moccia A, Medina Milanesi R. Qué saben las adolescentes acerca de los métodos anticonceptivos y cómo los usan: estudio en una población adolescente de Piedras Blancas. Rev Méd Urug 2006; 22:185-90. 
42. Manlove J, Ryan S, Franzetta K. Contraceptive use patterns across teens' sexual relationships: the role of relationships, partners, and sexual histories. Demography 2007; 44:603-21.

43. Vázquez A. Experiencia subjetiva del tiempo y su influencia en el comportamiento: revisión y modelos. Psicol Teor Pesq 2011; 27:215-23.

44. Rodriguez J. Reproducción en la adolescencia en América Latina y el Caribe: ¿Una anomalía a escala mundial? In: Anales del III Congreso de la Asociación Latinoamericana de Población. http://www.alapop.org/alap/images/ DOCSFINAIS_PDF/ALAP_2008_FINAL_21. pdf (accedido el 05/Jul/2020).

45. Menezes GMS, Aquino EML, Silva DO. Induced abortion during youth: social inequalities in the outcome of the first pregnancy. Cad Saúde Pública 2006; 22:1431-46.

46. Sanger C. Decisional dignity: teenage abortion, bypass hearings, and the misuse of law. Columbia J Gend Law 2009; 18:409-99.

47. Domínguez B, Sánchez R, Rizo EM, Chacón MC. Comportamiento del aborto inducido en la adolescencia. Rev Cuba Obstet Ginecol 2010; 36:145-9.

48. Doblado I, De la Rosa I, Junco A. Aborto en la adolescencia un problema de salud. Rev Cuba Obstet Ginecol 2010; 36:409-21.

49. Urgellés S, Reyes E, Figueroa M, Bermúdez Y. Comportamiento sexual y aborto provocado en adolescentes y jóvenes de escuelas de educación superior. Rev Cuba Obstet Ginecol 2012; 38:549-57.

50. Maranhão TA, Gomes KRO, Barros IC. Fatores preditores do abortamento entre jovens com experiência obstétrica. Rev Bras Epidemiol 2016; 19:494-508.
51. Palomar Verea C. Maternidad: historia y cultura. Revista de Estudios de Género: La Ventana $2005 ; 22: 35-67$.

52. Lombardi A. Entre madre e hijas. Acerca de la opresión psicológica. Buenos Aires: Paidós; 1989.

53. Davies SL, Crosby RA, Diclemente RJ. Family influences on adolescent health. In: DiClemente RJ, Santelli JS, Crosby RA, editores. Adolescent health: understanding and preventing risk behaviors. Hoboken: Jossey-Bass/Wiley; 2009. p. 391-410.

54. Sumter S, Bokhorst C, Steinberg L, Westenberg P. The developmental pattern of resistance to peer influence in adolescence: will the teenager ever be able to resist? J Adolesc 2009; 32:1009-21.

55. López Gómez A. Profesionales de la salud y aborto. Posibilidades y resistencias a la incorporación del enfoque de derechos a las prácticas de los/as profesionales de la salud. In: Ramos S, compiladora. Investigación sobre aborto en América Latina y el Caribe. Una agenda renovada para informar política pública e incidencia. Buenos Aires: Centro de Estudios de Estado y Sociedad/Population Council/ Consorcio Latinoamericano contra el Aborto Inseguro; 2015. p. 57-77.

56. Kumar A, Hessini L, Mitchell EMH. Conceptualising abortion stigma. Cult Health Sex 2009; 11:625-39.

57. Norris A, Bessett D, Steinberg JR, Kavanaugh ML, De Zordo S, Becker D. Abortion stigma: a reconceptualization of constituents, causes, and consequences. Womens Health Issues 2011; 21(3 Suppl):S49-54. 
Abstract

The article presents the results of a study on the experiences of adolescents that decided to terminate a pregnancy in the context of legal abortion services in Montevideo, Uruguay, from 2016 to 2018. The main objective was to examine the decision-making process and access to legal abortion from the perspective of the adolescent girls themselves. This involved analyzing circumstances, motives, and persons that influenced the decision and the ways this was handled in the health services. A cross-sectional, exploratory, and descriptive study was designed, based on a qualitative methodology. The main technique used for collecting the information was a semi-structured interview with 14 adolescents 17 to 19 years of age who had voluntarily terminated a pregnancy in public healthcare services. The motives for the abortion reveal the weight of socially accepted reasons, incorporated as their own. The interviews showed that the adolescent's mother plays an important role as the reference for the decision. The adolescents reported that they had made the decision to have the abortion before going to the health service. The results highlight the positive role of a legal framework that enables voluntary abortion services. The results help understand the experiences of adolescents facing a difficult life decision, the family's role, particularly that of the adolescent's mother, the legal framework's place in the decision, the weight of stigma and moral sanctions, and expectations towards care in health services.

Adolescence; Abortion; Decision Making; Health Services

\section{Resumo}

O artigo apresenta os resultados de uma pesquisa, desenvolvida entre 2016 e 2018, sobre as experiências de adolescentes que decidiram interromper a sua gravidez nas unidades de saúde para aborto legal em Montevidéu (Uruguai). Tinha como objetivo principal analisar o processo de tomada de decisão e o acesso ao aborto legal, do ponto de vista das próprias adolescentes. Isso envolvia avaliar as circunstancias, motivações e personagens que influenciaram a tomada de decisão e a forma como se deu no sistema de saúde. Foi construído um estudo transversal do tipo exploratório e descritivo baseado numa metodologia qualitativa. A técnica principal utilizada para a coleta de informação era a entrevista semiestruturada; foram entrevistadas 14 adolescentes entre 17 e 19 anos de idade, que tinham interrompido voluntariamente uma gravidez no sistema público de saúde. Os motivos para abortar refletem o peso das razões socialmente aceitas, incorporadas como motivos próprios. Observou-se que a mãe desempenha um papel relevante, como referente na decisão. As adolescentes afirmaram ter tomado a decisão de abortar antes de se dirigir à unidade de saúde. Destaca-se o papel positivo da existência de um marco legal que habilita serviços de aborto voluntário. Os resultados contribuem para o entendimento dos processos vivenciados pelas adolescentes diante de uma decisão vital difícil, o papel da família, em especial da figura materna, o lugar ocupado pelo marco legal na tomada da decisão, o peso do estigma e da sanção moral, além das expectativas sobre o atendimento nas unidades de saúde.

Adolescência; Aborto; Tomada de Decisões;

Serviços de Saúde
Recibido el 14/Dic/2019

Versión final presentada el 05/Jul/2020

Aprobado el 15/Jul/2020 